FACTA UNIVERSITATIS (NIŠ)

Ser. Math. Inform. Vol. 34, No 5 (2019), 815-822

https://doi.org/10.22190/FUMI1905815M

\title{
ARENS REGULARITY AND STRONG IRREGULARITY OF CERTAIN BILINEAR MAPPINGS
}

\author{
Somayeh Mohammadzadeh and Sedigheh Barootkoob
}

(c) 2019 by University of Niš, Serbia | Creative Commons Licence: CC BY-NC-ND

Abstract. In this paper, the relations between the topological centers of bounded bilinear mappings and some of their higher rank adjoints are investigated. Particularly, for a Banach algebra $A$, some results about the Banach $A$-modules and Arens regularity and strong Arens irregularity of module actions will be obtained.

Keywords: Normed algebra, Arens regular, strongly irregular, bounded bilinear map, Banach module.

\section{Introduction}

Suppose that $f: X \times Y \longrightarrow Z$ is a bounded bilinear mapping on normed spaces $X, Y$ and $Z$ and let $X^{*}$ be the topological dual of $X$. The adjoint of $f$ is the bounded bilinear map $f^{*}: Z^{*} \times X \longrightarrow Y^{*}$ defined by

$$
\left\langle f^{*}\left(z^{*}, x\right), y\right\rangle=\left\langle z^{*}, f(x, y)\right\rangle \quad\left(x \in X, y \in Y, z^{*} \in Z^{*}\right) .
$$

Using this method, the higher rank adjoints of $f$ can be defined by setting $f^{* *}=$ $\left(f^{*}\right)^{*}$. This notion was first introduced by Arens [1]. The $n$th adjoint of $f$ will be denoted by $f^{(n)}$ for $n>3$.

The mapping $f^{t}$ will be considered as the bounded bilinear map from $Y \times X$ into $Z$ defined by $f^{t}(y, x)=f(x, y)$. The mapping $f$ has two extensions $f^{* * *}$ and $f^{t * * * t}$ on $X^{* *} \times Y^{* *}$. The topological centers of the map $f$ are defined as follows:

$Z_{\ell}(f):=\left\{x^{* *} \in X^{* *} ; f^{* * *}\left(x^{* *}, y^{* *}\right)=f^{t * * * t}\left(x^{* *}, y^{* *}\right)\right.$ for every $\left.y^{* *} \in Y^{* *}\right\}$ and $Z_{r}(f):=\left\{y^{* *} \in Y^{* *} ; f^{* * *}\left(x^{* *}, y^{* *}\right)=f^{t * * * t}\left(x^{* *}, y^{* *}\right)\right.$ for every $\left.x^{* *} \in X^{* *}\right\}$.

When $f$ is the product $\pi$ of a normed algebra $A, \pi^{* * *}$ and $\pi^{t * * * t}$ are really the first and second Arens products of $A^{* *}$ which will be denoted by $\square$ and $\diamond$, respectively. In

Received April 08, 2019; accepted August 14, 2019

2010 Mathematics Subject Classification. Primary 46H20; Secondary 46H25 
this case the above topological centers are denoted by $Z_{\ell}\left(A^{* *}\right)$ and $Z_{r}\left(A^{* *}\right)$, respectively. The mapping $f$ is called (Arens) regular when $f^{* * *}=f^{t * * * t}$ and the normed algebra $A$ is said to be (Arens) regular if its product mapping is regular. The bilinear mapping $f$ is said to be left (resp. right) strongly (Arens) irregular if $Z_{\ell}(f)=X$ (resp. $Z_{r}(f)=Y$ ). Let $A$ be a normed algebra and let $X$ be a normed $A$-module ,with module actions $\pi_{\ell}$ and $\pi_{r}$, denoted by $\left(\pi_{\ell}, X, \pi_{r}\right)$. Then $\left(\pi_{r}^{t * t}, X^{*}, \pi_{\ell}^{*}\right)$ is a normed $A$-module. Also, $\left(\pi_{\ell}^{* * *}, X^{* *}, \pi_{r}^{* * *}\right)$ and $\left(\pi_{\ell}^{t * * * t}, X^{* *}, \pi_{r}^{t * * t}\right)$ are normed $A^{* *}$-modules with first and second Arens products, respectively. For further details about these concepts one can refer to $[2,3,8]$.

A bounded linear mapping $D: A \longrightarrow X^{*}$ is said to be a derivation if $D(a b)=$ $\pi_{\ell}^{*}(D(a), b)+\pi_{r}^{t * t}(a, D(b))$ for all $a, b \in A$. The mapping $D$ is said to be an inner derivation if there exists $x^{*} \in X^{*}$ such that $D(a)=\pi_{\ell}^{*}\left(x^{*}, a\right)-\pi_{r}^{t * t}\left(a, x^{*}\right)$ for each $a \in A$. Impose some conditions on the normed algebra $A$ or the normed $A$-module $\left(\pi_{\ell}, X, \pi_{r}\right)$ implies that the bounded linear mapping $D^{* *}: A^{* *} \longrightarrow X^{* * *}$ is also a derivation. Some of these conditions have been demonstrated in [4] and [8].

In this paper, $A$ will be considered as a normed algebra with product $\pi$ and $X$, $Y, Z$ are assumed to be the normed spaces. In the first section, some conditions will be discussed under which the range of the third adjoint of the bounded bilinear mapping $f: X \times Y \longrightarrow Z$ lies in the weak closure of $Z$; see Theorem 2.1. Many of the results of this paper are obtained by this theorem. In section 2, we discuss some conditions under which a Banach algebra $A$ will be a (left or right) ideal in $A^{* *}$ and then we improve some results of [5]. In section 3, by using Theorem 2.1, we continue the studies of [8] about the second adjoint of derivations.

\section{Topological centers and their relations}

Suppose that $\bar{X}$ be the weak closure of normed space $X$ which is equal to its original closure.

Proposition 2.1. Let $f: X \times Y \longrightarrow Z$ be a bounded bilinear map. Then $\bar{X} \subseteq$ $Z_{\ell}(f)$ and $\bar{Y} \subseteq Z_{r}(f)$.

Proof. Let $x^{* *} \in \bar{X}$, then there is a net $\left\{x_{\alpha}\right\} \subseteq X$ which converges to $x^{* *}$ in weak topology of $X^{* *}$. Now for every $y^{* *} \in Y^{* *}$ and $z^{*} \in Z^{*}$ and bounded net $\left\{y_{\beta}\right\} \subseteq Y$, which is $w^{*}$-convergent to $y^{* *}$, we have:

$$
\begin{aligned}
\left\langle f^{t * * * t}\left(x^{* *}, y^{* *}\right), z^{*}\right\rangle & =\left\langle f^{t * * * *}\left(z^{*}, y^{* *}\right), x^{* *}\right\rangle \\
& =\lim _{\alpha}\left\langle f^{t * * * *}\left(z^{*}, y^{* *}\right), x_{\alpha}\right\rangle \\
& =\lim _{\alpha}\left\langle f^{t * * *}\left(y^{* *}, x_{\alpha}\right), z^{*}\right\rangle \\
& =\lim _{\alpha}\left\langle y^{* *}, f^{t * *}\left(x_{\alpha}, z^{*}\right)\right\rangle \\
& =\lim _{\alpha} \lim _{\beta}\left\langle f^{t * *}\left(x_{\alpha}, z^{*}\right), y_{\beta}\right\rangle
\end{aligned}
$$




$$
\begin{aligned}
& =\lim _{\alpha} \lim _{\beta}\left\langle f^{t *}\left(z^{*}, y_{\beta}\right), x_{\alpha}\right\rangle \\
& =\lim _{\alpha} \lim _{\beta}\left\langle z^{*}, f\left(x_{\alpha}, y_{\beta}\right)\right\rangle \\
& =\left\langle f^{* * *}\left(x^{* *}, y^{* *}\right), z^{*}\right\rangle .
\end{aligned}
$$

We thus have $x^{* *} \in Z_{\ell}(f)$; that is $\bar{X} \subseteq Z_{\ell}(f)$, as required. A similar proof may apply for the inclusion $\bar{Y} \subseteq Z_{r}(f)$.

Applying the above proposition for the multiplication of a normed algebra $A$ we have:

Corollary 2.1. For every normed algebra $A, \bar{A} \subseteq Z_{\ell}\left(A^{* *}\right) \cap Z_{r}\left(A^{* *}\right)$.

[2, Theorem 3.4] indicates some cases for which the inclusion relations in Proposition 2.1 are converted to the equality. In fact, for every approximately unital left and right normed $A$-modules $\left(\pi_{\ell}, X\right)$ and $\left(X, \pi_{r}\right)$,

$$
Z_{r}\left(\pi_{\ell}^{t *}\right)=\bar{X}=Z_{r}\left(\pi_{r}^{*}\right)
$$

whereas

$$
Z_{\ell}\left(\pi_{\ell}^{t *}\right)=X^{*}=Z_{\ell}\left(\pi_{r}^{*}\right)
$$

obviously, if $X$ is a Banach space, then $\pi_{\ell}^{t *}$ and $\pi_{r}^{*}$ are both strongly irregular. In this case the above conclusions will be trivial.

Theorem 2.1. Let $X, Y$ and $Z$ be normed spaces and let $f: X \times Y \longrightarrow Z$ be a bounded bilinear mapping. Then

(i) $f^{* * *}\left(x^{* *}, y^{* *}\right) \in \bar{Z}$, for every $x^{* *} \in Z_{r}\left(f^{*}\right)$ and $y^{* *} \in Z_{r}\left(f^{t *}\right)$;

(ii) $f^{* * *}\left(x, y^{* *}\right) \in \bar{Z}$, for every $x \in X$ and $y^{* *} \in Z_{r}\left(f^{t *}\right)$;

(iii) $f^{* * *}\left(x^{* *}, y\right) \in \bar{Z}$, for every $x^{* *} \in Z_{r}\left(f^{*}\right)$ and $y \in Y$.

Proof. Let $x^{* *} \in Z_{r}\left(f^{*}\right), y^{* *} \in Z_{r}\left(f^{t *}\right)$ and $z^{* * *} \in Z^{* * *}$. If $\left\{x_{\alpha}\right\},\left\{y_{\beta}\right\}$ and $\left\{z_{\gamma}^{*}\right\}$ be bounded nets which converge to these elements in $w^{*}$-topology, respectively, then

$$
\begin{aligned}
\left\langle z^{* * *}, f^{* * *}\left(x^{* *}, y^{* *}\right)\right\rangle & =\left\langle f^{* * * *}\left(z^{* * *}, x^{* *}\right), y^{* *}\right\rangle \\
& =\left\langle f^{* t * * * t}\left(z^{* * *}, x^{* *}\right), y^{* *}\right\rangle \\
& =\lim _{\alpha} \lim _{\gamma} \lim _{\beta}\left\langle f^{*}\left(z_{\gamma}^{*}, x_{\alpha}\right), y_{\beta}\right\rangle \\
& =\lim _{\alpha} \lim _{\gamma} \lim _{\beta}\left\langle f^{t *}\left(z_{\gamma}^{*}, y_{\beta}\right), x_{\alpha}\right\rangle \\
& =\lim _{\alpha} \lim _{\beta} \lim _{\gamma}\left\langle f^{t *}\left(z_{\gamma}^{*}, y_{\beta}\right), x_{\alpha}\right\rangle \\
& =\lim _{\alpha} \lim _{\beta} \lim _{\gamma}\left\langle f\left(x_{\alpha}, y_{\beta}\right), z_{\gamma}^{*}\right\rangle \\
& =\lim _{\alpha} \lim _{\beta}\left\langle z^{* * *}, f\left(x_{\alpha}, y_{\beta}\right)\right\rangle .
\end{aligned}
$$

Therefore $f^{* * *}\left(x^{* *}, y^{* *}\right)$ belongs to the weak closure of $Z$ which equals to its norm closure. Parts $(i i)$ and $($ iii) are direct results of part $(i)$ 
It is easy to show that $\left.f^{* * *}\right|_{X \times Y^{* *}}=f^{* t * t}$ and $\left.f^{* * *}\right|_{X^{* *} \times Y}=f^{t * t *}$. Consider the normed algebra $A$ and the normed $A$-module $\left(\pi_{\ell}, X, \pi_{r}\right)$. The following theorem reveals a relation between the topological center of such mappings and that of $f$.

Proposition 2.2. For every bounded bilinear mapping $f: X \times Y \longrightarrow Z$,

$$
Z_{\ell}\left(\left.f^{* * *}\right|_{X \times Y^{* *}}\right) \subseteq Z_{\ell}(f) \text { and } Z_{r}\left(\left.f^{* * *}\right|_{X^{* *} \times Y}\right) \subseteq Z_{r}(f) .
$$

Proof. We only prove the first inclusion. Let $x^{* *} \in Z_{\ell}\left(f^{* t * t}\right)$; then by Theorem 2.1

$$
f^{(4)}\left(z^{*}, x^{* *}\right) \in Y^{*} \quad\left(\forall z^{*} \in Z^{*}\right)
$$

and applying the proof of $\left[8\right.$, Theorem 2.1] implies that $x^{* *} \in Z_{\ell}(f)$.

As an straightforward consequence of the above theorem, the following corollary is obtained; although the part (iii) is a known result .

Corollary 2.2. Let $f: X \times Y \longrightarrow Z$ be a bounded bilinear mapping.

(i) If $f$ is left strongly irregular, then so does $\left.f^{* * *}\right|_{X \times Y^{* *}}$.

(ii) If $f$ is right strongly irregular, then so does $\left.f^{* * *}\right|_{X^{* *} \times Y}$.

(iii) If one of $\left.f^{* * *}\right|_{X \times Y^{* *}}$ or $\left.f^{* * *}\right|_{X^{* *} \times Y}$ are Arens regular, then $f$ is Arens regular.

Corollary 2.3. If $\pi$ is the product of a strongly irregular Banach algebra, then $\left.\pi^{* * *}\right|_{A \times A^{* *}}$ (resp. $\left.\left.\pi^{* * *}\right|_{A^{* *} \times A}\right)$ is left (resp. right) strongly irregular.

For example, the above corollary holds for the group algebras $L^{1}(G)$ and $M(G)$, as the known strongly irregular Banach algebras; see [6] and [7].

\section{3. $\quad X$ and $X^{*}$ as $A^{* *}$-modules}

Suppose that $A$ is an Arens regular Banach algebra and let $\left(\pi_{\ell}, X, \pi_{r}\right)$ be a Banach $A$-module. It has been shown in [8] that $\left(\pi_{r}^{* *}, X^{*}, \pi_{\ell}^{t * * t}\right)$ is a Banach $A^{* *}$-module if and only if for every $x \in X$, the bilinear mapping $\theta_{x}: A \times A \longrightarrow X$ defined by

$$
\theta_{x}(a, b)=\pi_{\ell}\left(a, \pi_{r}(x, b)\right)=\pi_{r}\left(\pi_{\ell}(a, x), b\right) \quad(a, b \in A)
$$

is regular. Now the following proposition as another application of Theorem 2.1, improves this result.

Proposition 3.1. Let $A$ be an Arens regular Banach algebra and let $\left(\pi_{\ell}, X, \pi_{r}\right)$ be a Banach $A$-module. If either $\pi_{r}^{t * t}$ or $\pi_{\ell}^{*}$ is regular, then $\left(\pi_{r}^{* *}, X^{*}, \pi_{\ell}^{t * * t}\right)$ is a Banach $A^{* *}$-module. 
Proof. Suppose that $x \in X$. The natural extensions of $\theta_{x}$ on $A^{* *} \times A^{* *}$ satisfies

$$
\begin{aligned}
\theta_{x}^{* * *}\left(a^{* *}, b^{* *}\right) & =\pi_{\ell}^{* * *}\left(a^{* *}, \pi_{r}^{* * *}\left(x, b^{* *}\right)\right)=\pi_{r}^{* * *}\left(\pi_{\ell}^{* * *}\left(a^{* *}, x\right), b^{* *}\right) \text { and } \\
\theta_{x}^{t * * * t}\left(a^{* *}, b^{* *}\right) & =\pi_{\ell}^{t * * * t}\left(a^{* *}, \pi_{r}^{t * * * t}\left(x, b^{* *}\right)\right)=\pi_{r}^{t * * * t}\left(\pi_{\ell}^{t * * * t}\left(a^{* *}, x\right), b^{* *}\right),
\end{aligned}
$$

for all $a^{* *}, b^{* *} \in A^{* *}$.

In the case that $\pi_{r}^{t * t}$ is regular, Theorem 2.1 implies that $\pi_{r}^{* * *}\left(x, b^{* *}\right) \in \bar{X}=X$, for every $b^{* *} \in A^{* *}$ and so the above equalities demonstrate the regularity of $\theta_{x}$. A similar argument may applies for the case that $\pi_{\ell}^{*}$ is regular.

Let $A$ be a Banach algebra and let $\left(\pi_{\ell}, X, \pi_{r}\right)$ be a Banach $A$-module. Theorem 2.1 implies that

$$
\begin{gathered}
\pi_{\ell}^{* * *}\left(Z_{r}\left(\pi_{\ell}^{*}\right), X\right) \subseteq X, \pi_{r}^{* * *}\left(X, Z_{\ell}\left(\pi_{r}^{t * t}\right)\right) \subseteq X \text { and } \\
\pi_{\ell}^{t * * * t}\left(Z_{r}\left(\pi_{\ell}^{*}\right), X\right) \subseteq X, \pi_{r}^{t * * * t}\left(X, Z_{\ell}\left(\pi_{r}^{t * t}\right)\right) \subseteq X .
\end{gathered}
$$

Therefore, we obtain the following proposition.

Proposition 3.2. let $X$ be a Banach $A$-module as above.

(i) If $\pi_{r}^{t * t}$ is regular, then $X$ is a right Banach $A^{* *}$-module with either Arens products of $A^{* *}$.

(ii) If $\pi_{\ell}^{*}$ is regular, then $X$ is a left Banach $A^{* *}$-module with either Arens products.

(iii) If both module actions $\pi_{r}^{t * t}$ and $\pi_{\ell}^{*}$ are regular, then $X$ is a Banach $A^{* *}$-module (with either Arens products).

For the special case $X=A$, this implies the next result.

Corollary 3.1. Suppose that $A$ is a Banach algebra.

(i) If $\pi^{t * t}$ is regular, then $A$ is a right ideal of $A^{* *}$.

(ii) If $\pi^{*}$ is regular, then $A$ is a left ideal of $A^{* *}$.

(iii) If both $\pi^{t * t}$ and $\pi^{*}$ are regular, then $A$ is an ideal of $A^{* *}$.

Let $A$ be a Banach algebra and let $X$ be a Banach $A$-module. We say that $X$ factors $A$ on the left (resp. right) if $\pi_{r}(X, A)=X$ (resp. $\left.\pi_{\ell}(A, X)=X\right)$. Some relationships between the factorization property and Arens regularity are stated in [2] and [5]. Proposition 3.3 and Theorem 3.1 from [5] are of these cases which together with Corollary 3.1 provide conditions for the Arens regularity of $A$.

Proposition 3.3. [5, Corollary 4.1] Let $A$ be a left ideal in $A^{* *}$.

(1) If $A^{*}$ factors $A$ on the right, then $A$ is Arens regular.

(2) If $A^{* *}$ factors $A$ on the right, then $Z_{A}\left(A^{* * *}\right)=A^{* * *}$.

As an immediate consequence of Corollary 3.1 and the part (1) of the above proposition we have the following result. 
Corollary 3.2. If $\pi^{*}$ is regular and $A^{*}$ factors $A$ on the right, then $A$ is Arens regular.

Theorem 3.1. [5, Theorem 4.2] Let $A$ be a right ideal in $A^{* *}$. In each of the following situations, $A$ is Arens regular.

(1) $A^{*}$ factors $A$ on the left.

(2) $A^{* *}$ factors $A$ on the left.

As another application of Corollary 3.1, the next result is obtained by applying the above theorem.

Corollary 3.3. If $\pi^{t * t}$ is regular and $A^{*}$ or $A^{* *}$ factors $A$ on the left, then $A$ is Arens regular.

\section{The second adjoint of a derivation}

The following result as a consequence of [4, Proposition 6.2] and Theorem 2.1 indicates some conditions under which the second adjoint of a derivation is a derivation too.

Proposition 4.1. Suppose that $A$ is a Banach algebra. If the mappings $\pi^{*}$ and $\pi^{t *}$ are both regular, then the second adjoint of every inner derivation $D: A \longrightarrow A^{*}$ is also a derivation.

Proof. As it was shown in [4, Proposition 6.2], the second adjoint of every inner derivation $D: A \longrightarrow A^{*}$ is a derivation if and only if

$$
\left(b^{* *} \square c^{* *}\right) \diamond a^{* *}+\left(c^{* *} \square a^{* *}\right) \diamond b^{* *}-c^{* *} \diamond\left(a^{* *} \square b^{* *}\right)-b^{* *} \square\left(c^{* *} \square a^{* *}\right)=0
$$

for every $a^{* *}, b^{* *}, c^{* *} \in A^{* *}$. Now if $\pi^{*}$ and $\pi^{t *}$ are regular, then Theorem 2.1 implies that

$$
\left(b^{* *} \square c^{* *}\right) \diamond a^{* *}=\left(b^{* *} \square c^{* *}\right) \square a^{* *}=b^{* *} \square\left(c^{* *} \square a^{* *}\right) ;
$$

and also

$$
\left(c^{* *} \square a^{* *}\right) \diamond b^{* *}=\left(c^{* *} \square a^{* *}\right) \square b^{* *}=c^{* *} \square\left(a^{* *} \square b^{* *}\right)=c^{* *} \diamond\left(a^{* *} \square b^{* *}\right) .
$$

These equalities complete the proof.

Let $\left(\pi_{\ell}, X, \pi_{r}\right)$ be a Banach $A$-module and let $D: A \longrightarrow X^{*}$ be a derivation. [8, Theorem 4.2] indicates that the second adjoint of $D$ is a derivation on $\left(A^{* *}, \square\right)$ (resp. $\left.\left(A^{* *}, \diamond\right)\right)$ iff $\pi_{r}^{* * * *}\left(D^{* *}\left(A^{* *}\right), X^{* *}\right) \subseteq A^{*}\left(\right.$ resp. $\left.\pi_{\ell}^{t * * * *}\left(D^{* *}\left(A^{* *}\right), X^{* *}\right) \subseteq A^{*}\right)$. This theorem together with Theorem 2.1, implies the following corollary. 
Corollary 4.1. By the above hypothesis,

(i) If $\pi_{r}^{* t *}$ is regular and $D^{* *}\left(A^{* *}\right) \subseteq Z_{r}\left(\pi_{r}^{* *}\right)$, then $D^{* *}$ is a derivation on $\left(A^{* *}, \square\right)$.

(ii) If $\pi_{\ell}^{t * t *}$ is regular and $D^{* *}\left(A^{* *}\right) \subseteq Z_{\ell}\left(\pi_{\ell}^{t * * t}\right)$, then $D^{* *}$ is a derivation on $\left(A^{* *}, \diamond\right)$.

Applying [8, Corollary 4.4] for the case $X=A$ reveals that $D^{* *}$ is a derivation on $\left(A^{* *}, \square\right)\left(\operatorname{resp} .\left(A^{* *}, \diamond\right)\right)$ if both $\pi$ and $\pi^{t *}$ (resp. $\left.\pi^{*}\right)$ are Arens regular. Now by using corollaries 3.2 and 3.3 we obtain the next result.

Corollary 4.2. Suppose that $A$ is a Banach algebra and $D: A \longrightarrow A^{*}$ is a derivation.

(i) If $\pi^{*}$ is regular and $A^{*}$ factors $A$ on the right, then $D^{* *}:\left(A^{* *}, \diamond\right) \longrightarrow A^{* * *}$ is a derivation.

(ii) If $\pi^{t * t}$ is regular and $A^{*}$ factors $A$ or $A^{* *}$ on the left, then $D^{* *}:\left(A^{* *}, \square\right) \longrightarrow$ $A^{* * *}$ is a derivation.

\section{REF EREN CES}

1. A. Arens: The adjoint of a bilinear operation. Proc. Amer. Math. Soc. 2 (1951), 839-848.

2. S. Barootкoob, S. Mohammadzadeh and H. R. E. Vishki: Topological centers of certain Banach module actions. Bull. Iranian Math. Soc. 35 (2009), no. 2, 25-36.

3. H. G. DAles: Banach Algebras and Automatic Continuity. London Math. Soc. , Monographs 24, Clarendon Press, Oxford, 2000.

4. H. G. Dales, A. Rodrigues-Palacios and M. V. Velasco: The second transpose of a derivation. J. London Math. Soc. 64 (2001), no. 2, 707-721.

5. M. Eshaghi Gordgi and M. Filali: Arens regularity of module actions. Studia Math. 181 (2007), no. 3, 237-254.

6. A. T. LAU and V. LOSERT: On the second conjugate algebra of a locally compact group. J. London Math. Soc. 37 (1988), 464-470.

7. V. Losert, M. Neufang, J. Pachl and J. Steprāns: proof of the Ghahramani- Lau conjecture. Advances in Mathematics 290 (2016), 709 - 738.

8. S. Mohammadzadeh and H. R. E. Vishki: Arens regularity of module actions and the second adjoint of a derivation. Bull. Austral. Math. Soc. 77 (2008), 465-476.

Somayeh Mohammadzadeh

Mathematics Department

Faculty of Science

University of Bojnord

P. O. Box 1339

Bojnord, Iran

smohamad@ub.ac.ir 
Sedigheh Barootkoob

Mathematics Department

Faculty of Science

University of Bojnord

P. O. Box 1339

Bojnord, Iran

s.barutkub@ub.ac.ir 